\title{
From Polychemotherapy Regimens to Molecular Targeting Strategies
}

\author{
Cyrus Chargari
}

$\mathrm{N}^{2}$ asopharyngeal carcinoma (NPC) has a very low prevalence in Europe or in the United States. On the contrary, it is a significant health care issue in Asia with an incidence about 100-fold higher compared with other populations. Underlying explanations include genetic predisposition, environmental factors, and/or infection with Epstein-Barr virus (EBV). Consequently, Asian teams have been particularly involved in the fundamental and clinical research on this disease, which differs from other head and neck carcinomas in risk factors, natural history, histology, and response to anticancer therapies such as chemotherapy or radiation therapy. A search on Clinicaltrial.gov in August 2013 using the search term "nasopharyngeal carcinoma" identified 110 recruiting studies. East Asia was the most involved geographic region with 56 studies, compared with 41 clinical trials for North America and only 8 for Europe.

As recently reviewed by Lee and colleagues, lifestyle changes have radically decreased the incidence of tumor during past three decades. In parallel, there has been substantial amount of efforts to optimize therapeutics for locally advanced NPC through new irradiation devices, such as intensity-modulated radiation therapy, which have enhanced locoregional control while decreasing treatment-related toxicity. ${ }^{[1]}$ However, the prognosis of metastatic disease remains dismal, with overall survival being approximately 12 months. Numerous systemic agents have been tested, but a few have shown improvement compared to the standard cisplatin-based chemotherapy regimen, which yields the best antitumor effects. ${ }^{[2]}$ There is clearly a need to investigate new treatments in metastatic NPC.

In this issue of Biomedical Journal, Chia-Hsun Hsieh and coauthors report results from a phase II study combining cisplatin, oral tegafur-uracil, leucovorin, and mitomycin C in patients with recurrent or metastatic non-keratinizing or undifferentiated NPC. ${ }^{[3]}$ Response rates and survival times reported by the authors compare favorably to data obtained with cisplatin-based combinations from the literature. This study shows that there is still a substantial place for conventional systemic chemotherapeutics, based on the rationale that each drug targets a mechanism of tumor pro- gression (cell cycle, hypoxia, DNA repair). With adequate agents at relatively well-tolerated doses, polychemotherapy regimens may yield an acceptable efficacy/toxicity ratio. However, long-term survival remains to be improved and optimization of tomorrow's treatment will probably incorporate molecular targeted agents.

Several biological pathways are being investigated in metastatic NPC, alone or in combination with conventional chemotherapy. Epidermal Growth Factor Receptor (EGFR) has been the most studied target in head and neck carcinoma. Its overexpression correlates with poorer prognosis and more frequent locoregional relapses. In the randomized study EXTREME, adding anti-EGFR monoclonal antibody cetuximab to platinum-based chemotherapy plus fluorouracil alone improved overall survival as the first-line treatment in patients with recurrent or metastatic head and neck squamous cell carcinoma. However, NPC patients were not eligible to study enrollment. ${ }^{[4]}$ Despite the rationale of targeting EGFR in NPC, cetuximab has not drastically improved the clinical outcome in early phase trials focusing on this tumor site. Median time to progression (TTP) was about 3 months in the phase II study published by Chan and colleagues in $2005 .^{[5]}$ It is noteworthy that patients included in this study had EGFR-expressing tumors, but were heavily pretreated patients who had previously experienced treatment failure while receiving platinum-based chemotherapy. Moreover, TTP was longer in patients with a confirmed response (about 6 months). ${ }^{[5]}$ Another potential tumor target is neoangiogenesis. Recently, combination of tyrosine kinase inhibitor (TKI) sorafenib (inhibitor of vascular endothelial growth factor receptor (VEGFR), platelet-derived growth factor receptor (PDGFR), and RAF kinases) with standards cisplatin and 5-fluorouracil was shown tolerable and feasible in chemotherapy-naive NPC patients. Median progression-free survival time was 7.2 months $(95 \% \mathrm{CI}$ : 6.8-8.4 months), justifying further randomized comparison. ${ }^{[6]}$ Multitargeted receptor TKI pazopanib (inhibitor of PDGFR, c-KIT, and VEGFR1, 2, 3) has shown promising activity in phase II study in Asian patients with recurrent or metastatic NPC. ${ }^{[7]}$ Another point is that the biological

From the Department of Radiation Oncology, Val-de-Grâce Hospital, Paris, France

Received: Aug. 12, 2013; Accepted: Aug. 28, 2013

Correspondence to: Dr. Cyrus Chargari, Department of Radiation Oncology, Val-de-Grâce Military Hospital, Paris, France. Tel: 33-1-40-514558; Fax: 33-1-40-514670; E-mail: chargari-vdg@ hotmail.fr

DOI: $10.4103 / 2319-4170.119702$ 
specificities of NPC provide opportunities for innovative therapeutic interventions. Thus, the contribution of EBV to NPC encourages strategies modulating antitumor immunity, for example, through EBV-specific cytotoxic cells or adenoviral vector-based vaccine. ${ }^{[8,9]}$ Encouraging results have been reported in early phase trials, but a randomized confirmation is still lacking. Finally, numerous molecular mechanisms involved in EBV-mediated tumorigenesis could be specifically targeted, such as deregulated molecular pathways involved in cell proliferation, hypoxia, inhibition of apoptosis, or tumor cell invasion. ${ }^{[10-12]}$

There is no doubt that good knowledge of tumor biology will allow new therapeutic strategies to emerge. However, costs of drug developments may limit the investigations in the field of novel pharmacological targeted agents, particularly in the situation of economic crisis. Tumor types that are not considered as "high-priority" health care issues because of their rarity are particularly at risk. Further improvements should be based on a strong preclinical rationale in the context of translational research. Incorporation of biomarkers for treatment response should also be part of these developments. This is the prerequisite for optimizing clinical development of targeted agents and thus avoiding costly and disappointing clinical studies.

\section{REFERENCES}

1. Lee AW, Ng WT, Chan YH, Sze H, Chan C, Lam TH. The battle against nasopharyngeal cancer. Radiother Oncol 2012;104:272-8.

2. Chan AT. Nasopharyngeal carcinoma. Annals Oncol 2010;21:308-12.
3. Hsieh $\mathrm{CH}$, Hsu CL, Wang CH, Liaw CC, Chen JS, Chang HK, et al. Cisplatin, tegafur-uracil and leucovorin plus mitomycin C: An acceptably effective and toxic regimen for patients with recurrent or metastatic nasopharyngeal carcinoma. Biomed J 2013;36:229-36.

4. Vermorken JB, Mesia R, Rivera F, Remenar E, Kawecki A, Rottey S, et al. Platinum-based chemotherapy plus cetuximab in head and neck cancer. N Engl J Med 2008;359:1116-27.

5. Chan AT, Hsu MM, Goh BC, Hui EP, Liu TW, Millward MJ, et al. Multicenter, phase II study of cetuximab in combination with carboplatin in patients with recurrent or metastatic nasopharyngeal carcinoma. J Clin Oncol 2005;23:3568-76.

6. Xue C, Huang Y, Huang PY, Yu QT, Pan JJ, Liu LZ, et al. Phase II study of sorafenib in combination with cisplatin and 5-fluorouracil to treat recurrent or metastatic nasopharyngeal carcinoma. Ann Oncol 2013;24:1055-61.

7. Lim WT, Ng QS, Ivy P, Leong SS, Singh O, Chowbay B, et al. A Phase II study of pazopanib in Asian patients with recurrent/metastatic nasopharyngeal carcinoma. Clin Cancer Res 2011;17:5481-9.

8. Smith C, Tsang J, Beagley L, Chua D, Lee V, Li V, et al. Effective treatment of metastatic forms of Epstein-Barr virus-associated nasopharyngeal carcinoma with a novel adenovirus-based adoptive immunotherapy. Cancer Res 2012;72:1116-25.

9. Chua D, Huang J, Zheng B, Lau SY, Luk W, Kwong DL, et al. Adoptive transfer of autologous Epstein-Barr virus-specific cytotoxic T cells for nasopharyngeal carcinoma. Int J Cancer 2001;94:73-80.

10. Chou J, Lin YC, Kim J, You L, Xu Z, He B, et al. Nasopharyngeal carcinoma--review of the molecular mechanisms of tumorigenesis. Head Neck 2008;30:946-63.

11. Zhang L, Chen QY, Liu H, Tang LQ, Mai HQ. Emerging treatment options for nasopharyngeal carcinoma. Drug Des Devel Ther 2013;7:37-52.

12. Chan SL, Ma BB. Novel systemic therapeutic for nasopharyngeal carcinoma. Expert Opin Ther Targets 2012;16:63-8. 